\title{
Disposiciones, competencias cognitivas y suerte epistémica
}

Modesto M. Gómez-Alonso

Universidad Pontificia de Salamanca modestomga@hotmail.com

No sería incorrecto señalar que los dos problemas que más relevancia han tenido en la configuración de la agenda epistemológica posterior a Gettier han sido el de la suerte epistémica y el del principio de cierre.

Lo que los casos de Gettier subrayaron es la incompatibilidad de suerte y conocimiento, el hecho de que no atribuimos conocimiento a un sujeto cuando ha alcanzado la verdad de modo fortuito; es decir, cuando, en la medida en que las razones de $S$ a favor de $P$ dejan indeterminado su valor de verdad, constatamos que el agente podría fácilmente haber contado con las mismas razones, y que su creencia fuese falsa (ver Dancy, 1985: 134). Con ello, inauguraban un programa cuyo objeto es identificar cláusulas que, asegurando la verdad de la creencia, determinen condiciones suficientes de conocimiento. Teniendo en cuenta que la suerte que se opone al conocimiento se define a partir de criterios robustos, se trata de un programa especialmente estricto. Tal como muestran los ejemplos de la lotería inversa y de los graneros falsos, bastaría con que el sujeto, disponiendo de las mismas evidencias, pudiese haberse 
equivocado, para que la verdad de su creencia fuese accidental (y su creencia no contase como conocimiento): es, por tanto, suficiente un único defeater (ver Klein, 1981: 146) para que un contexto se encuentre epistémicamente contaminado.

Por otra parte, el principio de cierre (la validez lógica del condicional «Si $S$ sabe que $P$-donde $P$ es una proposición empírica- entonces $S$ sabe que $\neg H$-donde $H$ es una hipótesis escéptica global: el cerebro en la probeta, el Dios engañador cartesiano, el Inhabilitador de Sosa...-) forzaba a las concepciones internistas del conocimiento (que definen éste en virtud de la capacidad del sujeto de defender sus creencias in foro interno, esto es, del peso de las razones o evidencias a las que éste tiene acceso) a afrontar un dilema inaceptable: entre la eliminación (contraintuitiva) de dicho principio o la aceptación (epistémicamente inasumible) del escepticismo radical que, si los escenarios escépticos globales son irrefutables, se sigue de esa concepción. No es de extrañar, por ello, que con el fin de preservar el principio de cierre sin desembocar en el escepticismo, para evitar el dilema internista, se recusase al completo la concepción del conocimiento que lo genera, y que se definiese éste en términos de cláusulas externistas. Si para que sea verdad que el sujeto sabe no es necesario que sepa que sabe (que posea conocimiento reflexivo), bastará con el hecho bruto de que la creencia cumpla determinadas condiciones externas (aunque el sujeto ignore que se cumplen) para que el agente sepa que $P, \mathrm{y}$, en consecuencia, para que sepa (aunque no lo sepa reflexivamente) que no es un cerebro en una probeta ${ }^{1}$.

La conjunción de ambos factores configura una agenda centrada en la búsqueda de condiciones externas que asegu-

1 Se trata de una estrategia neo-mooreana, que, sin embargo, se desarrolla dentro de un modelo epistémico opuesto al del internismo de Moore. 
ren modalmente la verdad de una creencia (de forma que ésta no hubiese podido ser falsa fácilmente, o, lo que es igual, que no hubiese podido ser falsa en situaciones contrafácticas próximas), una agenda que ha dominado el panorama epistemológico analítico reciente.

El criterio de seguridad se ciñe a esa agenda. Dicho criterio delimita el área de proximidad modal (o de situaciones relevantes para la determinación de si $S$ sabe) a partir del procedimiento empleado por el sujeto para adquirir su creencia, pero señala que es lo que suceda dentro de esta área, algo independiente del procedimiento empleado, lo que carga con el peso del conocimiento: sea cual fuese el método de adquisición de creencias (y su calidad epistémica), basta con que se produzcan aciertos en la totalidad de los escenarios próximos cuyo límite marca para que el sujeto sepa. Basta, por ello, con que la verdad se encuentre externamente asegurada (por ejemplo, porque el escenario es afortunado, y no existe una sola maqueta entre los graneros a los que el sujeto tiene acceso) para que podamos atribuir conocimiento.

Sin embargo, del hecho de que que las circunstancias modales próximas sean las apropiadas sea condición necesaria para saber, no se sigue que sea condición suficiente. Los criterios que garantizan la verdad de una creencia no aseguran, por sí solos, que se trate de conocimiento. Que, dadas las circunstancias, el sujeto no pueda fallar no significa que sepa. ¿Por qué? ¿Qué otra cláusula ha de cumplirse?

\section{II}

Desde su formulación seminal en el artículo de Ernest Sosa, «The Raft and the Pyramid» (1980: 3-25), el rasgo común a las diversas variedades de epistemología de virtudes es la defini- 
ción del conocimiento en términos de creencias basadas en el ejercicio de competencias cognitivas fiables asentadas en el sujeto, de modo que esta cláusula sea, como poco, una condición necesaria para su posesión. Esta corriente se desarrolla en contraposición al criterio de seguridad, cuyas deficiencias explota. Son, al menos, tres las razones que la legitiman: (i) En primer lugar, el hecho de que el criterio de seguridad no parece capaz de excluir la suerte. Al fin y al cabo que las circunstancias (actuales o modales) sean afortunadas es eso: una cuestión de buena fortuna. (ii) La constatación de que la mera acumulación de aciertos no basta para atribuir conocimiento a alguien, menos aún, si éstos se basan en métodos espúreos de adquisición de creencias (la adivinación, por ejemplo). En este sentido, lo que se subraya es una debilidad constitutiva del criterio de seguridad, que acomodaría ejemplos de creencias deficientemente formadas. (iii) La apreciación de un hecho relevante en las adscripciones ordinarias de conocimiento: el que éste se trate de un logro atribuible al sujeto epistémico, cosa que lo distingue de la mera adquisición de creencias verdaderas. Para que alguien sepa no basta con que el escenario sea tal que la verdad se encuentre externamente asegurada (por ejemplo, en virtud de «genios benignos» $\mathrm{O}$ «ángeles protectores» que enmascaren la incompetencia del agente, haciendo que éste, y con independencia de lo que haga, siempre acierte). El sujeto ha de poder responsabilizarse de su creencia, ha de poder ser sujeto de mérito epistémico. Lo que equivale a reclamar su agencia.

No es el objetivo de este trabajo desarrollar las diversas concepciones que se dan cita bajo el rótulo genérico de «epistemología de virtudes»; lo que sí me interesa subrayar es que sus versiones «mínimas» (aquéllas, como la defendida por Alvin Goldman, que definen el conocimiento en virtud de 
la fiabilidad de la competencia ejercida en la adquisición de una creencia más el acierto de ésta) no son inmunes a variaciones de los casos de Gettier. Es Sosa quien nos ofrece un contra-ejemplo significativo (2011: 67-18). S, cuya capacidad visual es fiable, asiste a una fiesta en el piso de un amigo. Allí, en base al ejercicio de su visión, forma la creencia de que determinada celebridad se encuentra en la habitación. Su creencia se debe a esta competencia fiable, y es verdadera; sin embargo, lo que $S$ ha visto no ha sido a la celebridad, sino un holograma suyo de tamaño natural. ¿Sabe $S$ que la celebridad se encuentra en la habitación? Intuitivamente, nuestra respuesta es negativa; de lo que se deduce que, porque el sujeto pudo haber fallado fácilmente ejerciendo una competencia (en general) fiable, el fiabilismo es incapaz de fijar las cláusulas que definen el conocimiento.

Casos como el anterior, que muestran la necesidad de corregir el mero fiabilismo, han justificado la versión de epistemología de virtudes defendida por Sosa en los dos volúmenes (2007 y 2009) de Apt Belief and Reflective Knowledge, una versión a la que denominaremos, de acuerdo con la terminología que el propio Sosa emplea en su producción más reciente, «teoría de la mera aptitud» (Sosa, 2011: 21). Los pilares de esta teoría son: (i) La caracterización del conocimiento como creencia apta, es decir, como creencia cuyo acierto manifiesta (o se explica en virtud de, o se debe) al ejercicio de una competencia ${ }^{2}$. Esta caracterización permite tanto diagnosticar por qué, en el caso de la celebridad, el sujeto no sabe (la competencia no explica la verdad de la creencia, sólo que el sujeto tenga esa

2 Se trata de creencias que instancian la estructura normativa AAA, acrónimo de accuracy (acierto), adroitness (destreza) y aptness (aptitud, esto es, acierto cuya causa es la competencia ejercida). 
creencia), cómo fijar un criterio sumamente plausible (nogettierizado) de conocimiento. (ii) La distinción entre conocimiento animal y conocimiento reflexivo, es decir, entre el hecho de que el sujeto posea conocimiento y el hecho de que disponga de acceso racional a lo que conoce, de que esté en posición de adscribirse conocimiento a sí mismo. Con ello, parecen poder conciliarse las intuiciones externistas y las internistas, de forma que, aunque el criterio de aptitud (referido a las creencias animales o de primer orden) se cumple (o incumple) con independencia de que el sujeto tenga acceso reflexivo a ello (sepa si sabe), este acceso es un bien epistémico.

Sin embargo, y tal como el propio Sosa ha subrayado en su último libro publicado: Knowing Full Well, dos problemas presionan a las teorías de la mera aptitud. Por un lado, el de la función del conocimiento reflexivo ( $\mathrm{y}$, por tanto, el del papel epistémico del sujeto), aspectos que la bi-compartimentación estricta y la consiguiente definición del conocimiento animal en términos puramente externistas (o de mera aptitud) o no explica o transforma en «extras» epistémicos, en elementos que pueden acompañar al conocimiento, pero que no son ni suficientes ni necesarios para saber. Por otra parte, el de si la aptitud, además de condición necesaria, es condición suficiente de conocimiento. Lo que da origen a este último problema es la existencia de casos donde el sujeto pudo haber fallado fácilmente, siendo apta su creencia; es decir, donde, aunque la competencia ejercida por el sujeto explica la verdad de su creencia, somos reticentes a atribuirle conocimiento. Se trata de ejemplos que gettierizan la teoría de la mera aptitud. Son estos casos los que me interesa destacar especialmente. 


\section{III}

Las competencias cognitivas (capacidad visual, memoria, racionalidad) a las que apela la epistemología de virtudes son habilidades. Las habilidades son, a su vez, un tipo especial de propiedades disposicionales, análogas a los ejemplos paradigmáticos de disposiciones: la fragilidad y la solubilidad. Las disposiciones se encuentran asociadas a una serie de condicionales de las que forman parte condiciones antecedentes desencadenantes y manifestaciones resultantes. Al mismo tiempo, presuponen condiciones apropiadas de manifestación, de forma que el hecho de que en condiciones inapropiadas no se manifieste una disposición no implica su falsación.

¿Cuál es el alcance de estas condiciones apropiadas? ¿Hasta dónde se extienden, modal o circunstancialmente, las condiciones que delimitan una manifestación apropiada? Lo que parece claro es que una condición básica de la manifestación de propiedades disposicionales de sentido común es que, para producirse ésta, nunca es necesario que las condiciones antecedentes desencadenen la manifestación resultante, no sólo en los mismos lugar, tiempo y circunstancias en los que el portador de la disposición se encuentre adecuadamente ubicado, sino también a lo largo de un espectro (actual o modal) que incluya una variedad amplia de lugares, tiempos y circunstancias adyacentes. En otras palabras, las condiciones apropiadas para la manifestación de una disposición carecen de extensión o alcance modal: las situaciones contrafácticas próximas no la afectan. Veamos dos ejemplos: al dejar caer un vaso de cristal sobre una superficie dura, el hecho de que se haga añicos manifiesta su fragilidad, por mucho que todas las superficies cercanas, por ser blandas, hubiesen impedido la manifestación de esa disposición en áreas próximas. De igual modo, que un terrón de azúcar se 
disuelva en un líquido caliente manifiesta su solubilidad, aunque un agente externo pudiese haber impedido la manifestación de esa disposición o la impidiese de hecho en la totalidad de ejemplos adyacentes (por ejemplo, y tal como sucede en el escenario del mago protector y de las disposiciones enmascaradas, porque dicho agente tiene el poder de congelar el líquido antes de que se disuelva el azúcar) (ver Johnston, 1992: 221-263).

Apliquemos la cláusula anterior al escenario (epistémicamente contaminado) de los graneros. Desconociendo que algunos de los supuestos graneros son realmente maquetas de cartón piedra, Barney para su coche frente a un granero real, y le señala a su hijo que eso es un granero. La creencia de Barney es verdadera. También es competente: el origen de esta creencia es el ejercicio, por parte del sujeto, de una competencia fiable (su capacidad visual). Pero, además, es apta: la verdad de la creencia manifiesta la competencia ejercida, de forma que es ésta la que explica por qué Barney ha acertado. Del mismo modo que del hecho de que la solubilidad del azúcar pudiese no haberse manifestado no se sigue que no se haya manifestado, del hecho de que la competencia de Barney pudiese fácilmente no haberse manifestado (en el supuesto de que hubiese señalado una maqueta: la falsedad de la creencia conlleva su falta de aptitud) no se sigue que, cuando lo que señala es un granero, no se haya manifestado. Por tanto, la creencia es apta aunque el escenario epistémico se encuentre contaminado. Y es precisamente esto lo que da pie al problema.

Se trata de un verdadero dilema, en el que las dos alternativas son igualmente inaceptables. Por un lado, el hecho de que las competencias sean un ejemplo más de disposiciones, y de que, por ello, puedan manifestarse aunque las circunstancias próximas (actuales o modales) sean adversas, se sigue que una creencia puede ser apta aunque no sea segura, esto 
es, que el hecho de que el sujeto pudiese haber fallado fácilmente no cancela el hecho de que, actualmente, su acierto manifieste su competencia. Por otro lado, la proximidad de escenarios adversos (la cláusula de seguridad) muestra que el acierto ha sido fortuito, $y$, por consiguiente, que su creencia no equivale a conocimiento. De este modo, parecemos obligados a optar entre dos únicas alternativas: o la creencia apta es conocimiento (cosa que atenta contra nuestras intuiciones fundamentales), o, porque no lo es, deberíamos definir el conocimiento en términos de aptitud más seguridad, de forma que el conocimiento dependa, después de todo, de circunstancias externas al sujeto, y que éste bien puede ignorar. Los problemas de esta segunda opción resultan obvios. Al tratarse de una variable incontrolable, la cláusula independiente de seguridad reintroduce la suerte y cancela el mérito del agente epistémico. En este nuevo formato, los mecanismos (o condiciones) externos que aseguran la verdad de la creencia no corrigen la incompetencia del agente, pero suplementan su aptitud. Aquí, la función del «ángel protector» no es ni reubicar la trayectoria de la creencia del agente ni reubicar el blanco para que acierte, sino limpiar el escenario de forma que el sujeto no pueda ser competente y fallar: en este sentido, las circunstancias no explican el acierto, pero sí el conocimiento. Sin embargo, el problema no es qué función tengan las circunstancias próximas (o, metafóricamente, cuál sea la función del «ángel protector»), sino que tengan una función. Lo que necesitamos es una teoría que incluya la seguridad sin excluir que el conocimiento sea un logro atribuible al sujeto. El logro de éste no puede reducirse a la adquisición de creencias verdaderas que, por sí mismas, no alcancen el estatus de saber. 


\section{IV}

Sosa no sólo proporciona esa concepción, sino que, gracias a ella, resuelve al mismo tiempo los dos problemas que presionan a las teorías de la mera aptitud: el de la función del conocimiento reflexivo, y el de la preservación de un marco epistemológico de competencia, acierto y aptitud, pese a que la aptitud no equivalga a conocimiento. Se trata de dilucidar nuestra intuición negativa, de explicar por qué rehusamos adscribir conocimiento a $S$ pese a que su creencia sea apta. Esto remite a la noción (reflexiva) de meta-aptitud. Lo que las circunstancias desfavorables próximas bloquean no es la aptitud de la creencia, sino el acceso (apropiado) del sujeto a la aptitud de su creencia, o, lo que es igual, la aptitud de la metacompetencia que éste ejerce al atribuirse (a sí mismo) una creencia apta. Al asumir un punto de vista de tercera persona, lo que constatamos es que, dadas las circunstancias en las que se encuentra Barney (o víctimas análogas de escenarios contaminados), cuando éste se atribuye una creencia apta acierta por casualidad: fácilmente hubiese creído que su creencia de primer orden es apta sin que ésta lo fuese. De este modo, lo que hacemos al negar que sabe es negar que sabe que su creencia es apta: careciendo de conocimiento reflexivo el sujeto carece, sin más, de conocimiento. Lo que equivale tanto a abandonar la definición de conocimiento reflexivo como «saber que se sabe» (sustituyéndola por «saber que la creencia es apta» o «creer aptamente que la creencia de primer orden es apta»), como a eliminar el «conocimiento animal». Éste pasa a ser, en términos de Sosa, simple «Cognición animal» (Sosa, 2011: 93). El conocimiento humano es, tautológicamente, conocimiento reflexivo, o, lo que es igual, el conocimiento reflexivo pasa de ser una simple condición de adscripción de conocimiento a ser condición de adscripción y condi- 
ción de posesión de conocimiento. Sólo si la creencia de segundo orden del sujeto es apta posee este conocimiento.

Nótese: (i) que, para que una creencia apta $P$ cuente como conocimiento, ha de estar guiada por la meta-aptitud de $Q$, la creencia en que, dado que las circunstancias (internas y externas) que rodean una declaración cognitiva son apropiadas, el ejercicio de una determinada competencia animal rendirá aciertos (las creencias resultado de ese ejercicio serán aptas), y, por tanto, en que $P$ es (será) una creencia apta; (ii) que la meta-competencia ejercida reflexivamente (en la evaluación de la adecuación de las circunstancias) no es idéntica a la competencia de primer orden; y (iii) que las dos condiciones arriba exigidas se satisfacen: que la creencia reflexiva es apta equivale a señalar que el sujeto sabe que el escenario es seguro, condición que, aunque incluye el hecho de su seguridad, no se reduce a él: el sujeto ha de tener acceso a ese hecho.

La última señalización podría alentar una crítica: ¿no son los criterios de Sosa demasiado estrictos?, ¿no eleva en exceso los niveles de escrutinio, sugiriendo que, por ejemplo, y en la medida en que para saber que lo que señala es un granero Barney ha de saber que el escenario es seguro, éste no podrá saber lo primero si antes no comprueba que no hay maquetas, es más, si no desecha la mera posibilidad de que las haya?, ¿y no es lo anterior un argumento en contra de su concepción del conocimiento?

Lo sería, si eso fuese lo que defiende. Pero no lo es. Nótense dos cosas: (i) que, tanto si se encuentra como si no se encuentra contaminado el escenario, el sujeto ejerce idéntica meta-competencia en la evaluación de la situación; y (ii) que, al señalar que su creencia particular $P$ es apta (al atribuirse una creencia apta), el sujeto lo hace en base a su creencia de que las circunstancias son seguras, de forma que de «Todas 
las creencias resultado de tal competencia son aptas» deduce «Esta creencia (resultado de tal competencia) es apta». La segunda apreciación impide que se reproduzca a nivel reflexivo el dilema que desarrollamos a nivel animal, de modo que una creencia particular pueda ser meta-apta sin ser conocimiento reflexivo. Lo primero indica que la diferencia entre aptitud y no-aptitud de segundo orden no se debe a la incompetencia del agente (de forma que éste tuviese que haber hecho más de lo que hizo para saber que su creencia de primer orden es apta), sino a que en un caso su creencia reflexiva es fal$s a$, y no en el otro. $\mathrm{O}$, lo que es igual: la meta-competencia que se ejerce para saber consiste en no percibir indicios de una situación desafortunada o anómala, es decir, en un tipo de evaluación racional en la que el mero silencio de la razón, al explicar el acierto de la creencia de segundo orden, constituye su meta-aptitud. Igual que una creencia puede ser apta sin ser meta-apta, puede ser meta-apta sin ser meta-meta-apta. Lo que uno tenga que hacer para saber (no accidentalmente) que sabe que su creencia es apta es muy distinto de lo que ha de hacer para saber que es apta: la posibilidad de mejorar nuestra posición epistémica, y de apropiarnos reflexivamente mejor de nuestras creencias, no implica que no dispongamos de cierto grado de apropiación, y que nuestra posición epistémica, aunque mejorable, no sea positiva.

El propio Descartes suscribiría esta posición, que reconoce que una creencia puede ser epistémicamente incompleta (de forma que el sujeto no se encuentre completamente integrado con sus creencias, y que sea concebible la escisión entre lo que cree y la actitud racional que toma respecto a ello), sin que por eso sea reprochable o fallida. Así, se hace justicia tanto al proyecto epistemológico cartesiano como a las adscripciones ordinarias de conocimiento. 
El anti-reduccionismo de Sosa no es el menor de los atractivos de su epistemología. Y esos atractivos, en sí mismos y en contraste con teorías rivales, no son precisamente pocos.

Bibliografía

Dancy, J. (1985). Introduction to Contemporary Epistemology. Oxford: Blackwell.

Johnston, M. (1992). How to Speak of Colors. Philosophical Studies 68, 221-263.

Klein, P. (1981). Certainty: A Refutation of Scepticism. Brighton, Sussex: The Harvester Press.

Sosa, E. (1980). The Raft and the Pyramid: Coherence versus Foundations in the Theory of Knowledge. Midwest Studies in Philosophy 5, 3-25.

Sosa, E. (1980). Knowing Full Well. Princeton \& Oxford: Princeton University Press. 


\section{Resumen}

El hecho de que la verdad de una creencia pueda manifestar, en condiciones modales adversas, la competencia epistémica ejercida por el sujeto, pone en entredicho las teorías de la mera aptitud. La epistemología de virtudes se ve, así, obligada a incluir el criterio de seguridad, pero de tal modo que el conocimiento sea un logro atribuible al agente. En su producción más reciente, Ernest Sosa ha mostrado que, para saber, el sujeto ha de disponer de acceso apropiado a la aptitud de su creencia, o, lo que es igual, que sus actuaciones epistémicas de primer orden han de estar guiadas por un conocimiento reflexivo o de segundo orden para constituir conocimiento humano. Desarrollaremos y defenderemos esta concepción, mostrando que hace justicia tanto al proyecto epistemológico cartesiano como a las declaraciones ordinarias de conocimiento.

Palabras clave: Ernest Sosa, competencias cognitivas, conocimiento reflexivo, disposiciones, epistemología de virtudes, suerte epistémica.

\section{Abstract}

As a subset of dispositions, cognitive competences require no modal or neighbourhood robustness. This means that, insofar as a belief might be apt without being knowledge, we have a counterexample to the simple aptness view. The requirement of safety must be so included in a virtue epistemology, but in such a way that the insight that knowledge is an achievement of the agent is preserved. In his latest version of a virtue epistemology, Ernest Sosa has argued that, in order to know, the agent's proper access to the aptness of his beliefs is required, or, in other words, that human knowl- 
edge requires that the apt performance on the first order must be guided by knowledge on the second order that the first order performance would be apt. We aim at developing and vindicating this view, arguing that it tallies both with ordinary cognitive practices and with the Cartesian project.

Key Words: Ernest Sosa, cognitive competences, dispositions, epistemic luck, reflective knowledge, virtue epistemology. 
